\title{
Long Noncoding RNA MNX1 antisense RNA 1 Exerts Oncogenic Functions in Bladder Cancer by Regulating miR-218-5p/RAB1A Axis
}

\author{
Jun Wang, Huiwu Xing, Abdul Aziz Nikzad, Bowen Liu, Yu Zhang, Songchao Li, \\ Erwei Zhang, and Zhankui Jia \\ Department of Urology, The First Affiliated Hospital of Zhengzhou University, Zhengzhou, People's Republic of China
}

Received October 3, 2019; accepted December 9, 2019

\begin{abstract}
LncRNA MNX1 antisense RNA 1 (MNX1-AS1) is significantly overexpressed in patients with bladder cancer, suggesting that it might be associated with bladder cancer. However, the molecular mechanism of MNX1-AS1 in bladder cancer remained indistinct. To illustrate the role of MNX1-AS1 in bladder cancer, the gain- and loss-of-function experiments were conducted in bladder cancer cells. Reduced expression of MNX1-AS1 could suppress cell proliferation, migration, invasion, and epithelialmesenchymal transition in bladder cancer cells, whereas overexpression of MNX1-AS1 resulted in the opposite effects. Mechanistic analysis demonstrated that miR-218-5p was a direct target of RAB1A. MNX1-AS1 could competitively bind to miR-218-5p to regulate RAB1A expression in bladder cancer cells. Furthermore, in vivo experiments revealed that reduced expression of MNX1-AS1 inhibited tumor growth and metastasis.
\end{abstract}

Taken together, MNX1-AS1 functions as a sponge to miR-218$5 p$ to modulate RAB1A expression in bladder cancer, which suggests that MNX1-AS1 might serve as a novel therapeutic target and a novel biomarker for metastasis and prognosis in bladder cancer.

\section{SIGNIFICANCE STATEMENT}

Our study demonstrates that long noncoding RNA MNX1-AS1 promotes the initiation and progression of bladder cancer. MNX1-AS1 regulates RAB1A expression to promote proliferation, migration, invasion, and epithelial-mesenchymal transitions of bladder cancer cells via miR-218-5p, which contributes to the tumor growth and metastasis of bladder cancer. Collectively, these results suggest that MNX1-AS1 might serve as a potential biomarker for bladder cancer.

\section{Introduction}

Bladder cancer is the ninth most common malignancy worldwide, and the global incidence of bladder cancer is increasing (Bray et al., 2018; Wong et al., 2018). The main therapeutic methods include surgery, radiation, and chemotherapy (Ferro et al., 2019). However, 5-year survival rate is dissatisfying because of its high rates of metastasis and recurrence (Chen et al., 2012; Li et al., 2013; Ferro et al., 2019). Therefore, studying the molecular mechanism of bladder cancer proliferation and metastasis can provide guidance for the development of diagnosis and therapeutic strategies.

Long noncoding RNAs (lncRNAs) are crucial regulators of gene expression and play vital functions in various biologic processes (Wang and Chang, 2011). Alterations in the function of lncRNAs are associated with various human cancers

The authors declared that there is no conflict of interest, financial or otherwise in this study.

https://doi.org/10.1124/jpet.119.262949.
(Lv et al., 2017b; Matboli et al., 2019; Sassenberg et al., 2019), including bladder cancer (Ma et al., 2019). Moreover, gene networks have been found to regulate the formation, development, and metastasis of bladder cancer. LncRNA H19 affected the progression of bladder cancer by regulating miR-29b-3p (Lv et al., 2017a). LncRNA Growth Arrest Specific 5 suppressed the proliferation of bladder cancer cells by inhibiting enhancer of zeste 2 polycomb repressive complex 2 subunit on the transcriptional level (Wang et al., 2018). LncRNA transcription factor Dp-1 inhibited bladder cancer via inactivating signal transducer and activator of transcription 3 signaling (Ma et al., 2019). These indicate that lncRNAs are closely related to the pathogenesis of bladder cancer.

LncRNA MNX1-AS1 (NR_038835.1) is located at chromosome 7q36.3, with a length of 992 bp (Yang et al., 2018). Previous studies indicated that MNX1-AS1 could facilitate the progression of lung cancer (Liu et al., 2019a), colon adenocarcinoma (Ye et al., 2019), cervical cancer (Liu et al., 2019b), ovarian cancer (Lv et al., 2017b), and glioblastoma (Gao et al., 2019). However, the biologic role of MNX1-AS1 in bladder cancer remains unknown. MNX1-AS1 has been reported to act

ABBREVIATIONS: CCK-8, Cell Counting Kit-8; EMT, epithelial-mesenchymal transition; IHC, immunohistochemistry; IncRNA, long noncoding RNA; mTORC1, mammalian target of rapamycin complex 1; NC, negative control; PBST, PBS containing 0.05\% Tween-20; PCR, polymerase chain reaction; p-S6K1, phosphorylated S6 kinase 1; S6K1, S6 kinase 1; sh-NC, negative control shRNA; sh-MNX1-AS1, shRNA targeting MNX1-AS1; shRNA, short hairpin RNA; siRNA, small interfering RNA; MNX1-AS1: MNX1 antisense RNA 1; RAB1A: member RAS oncogene family; RAB1A-wt: RAB1A containing the wild-type binding site for miR-218-5p; RAB1A-mut: RAB1A containing the mutant binding site for miR-218-5p 
A

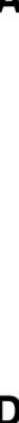

D
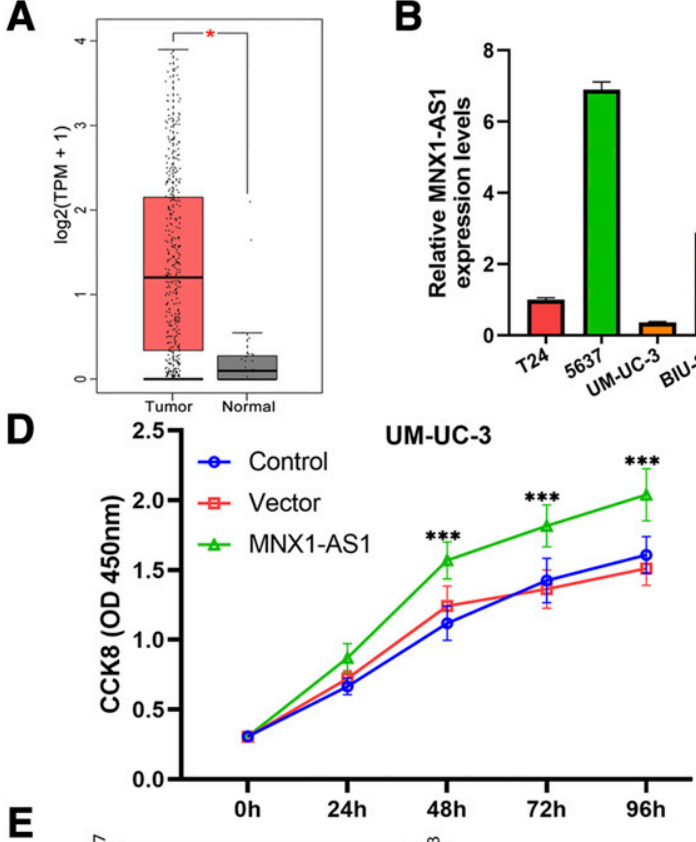

E
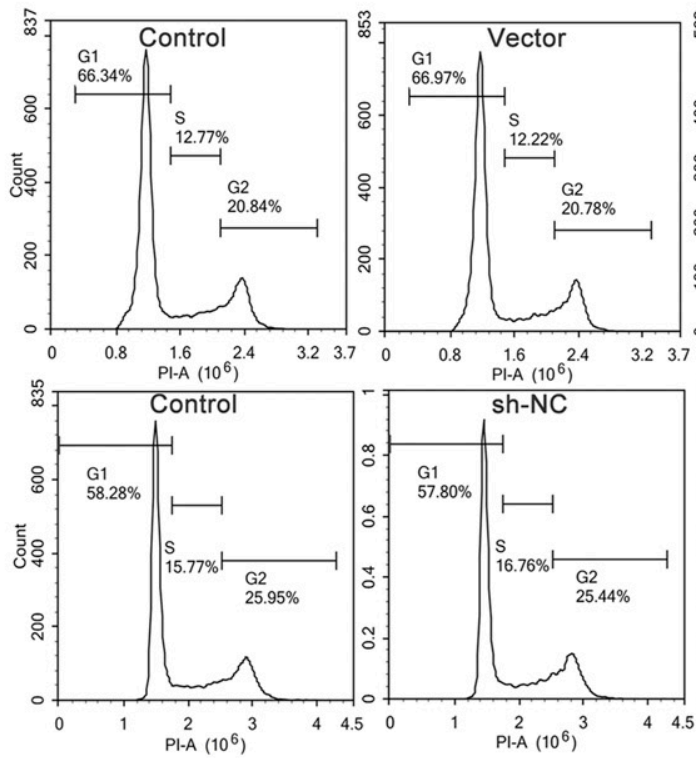

F
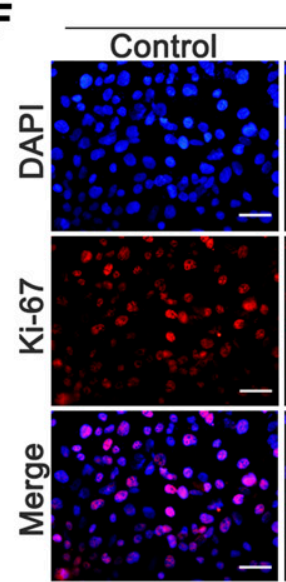

B

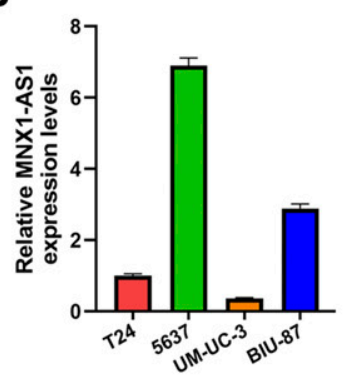

UM-UC-3

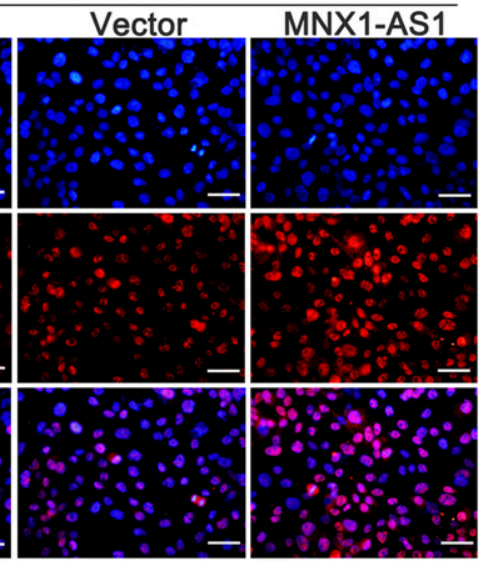

C

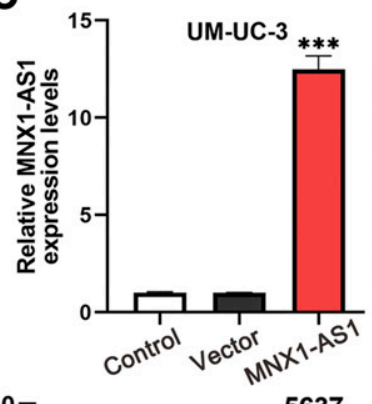

5637

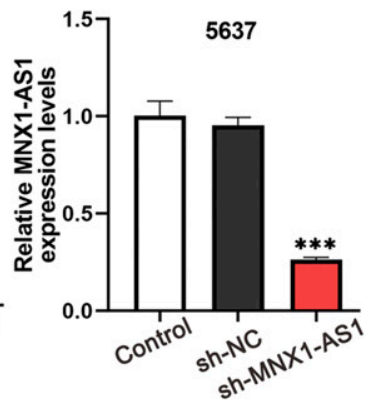

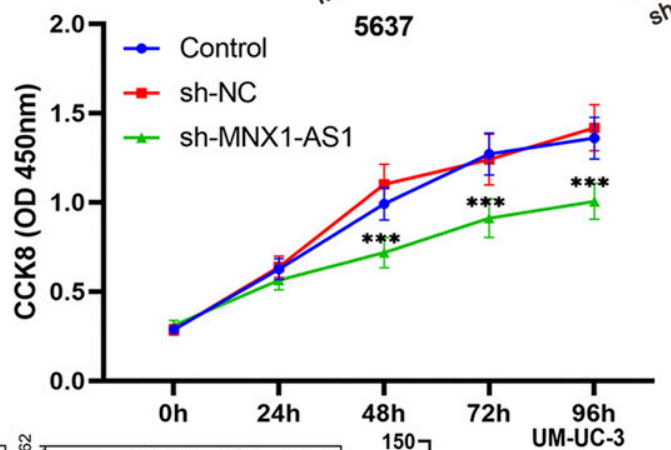
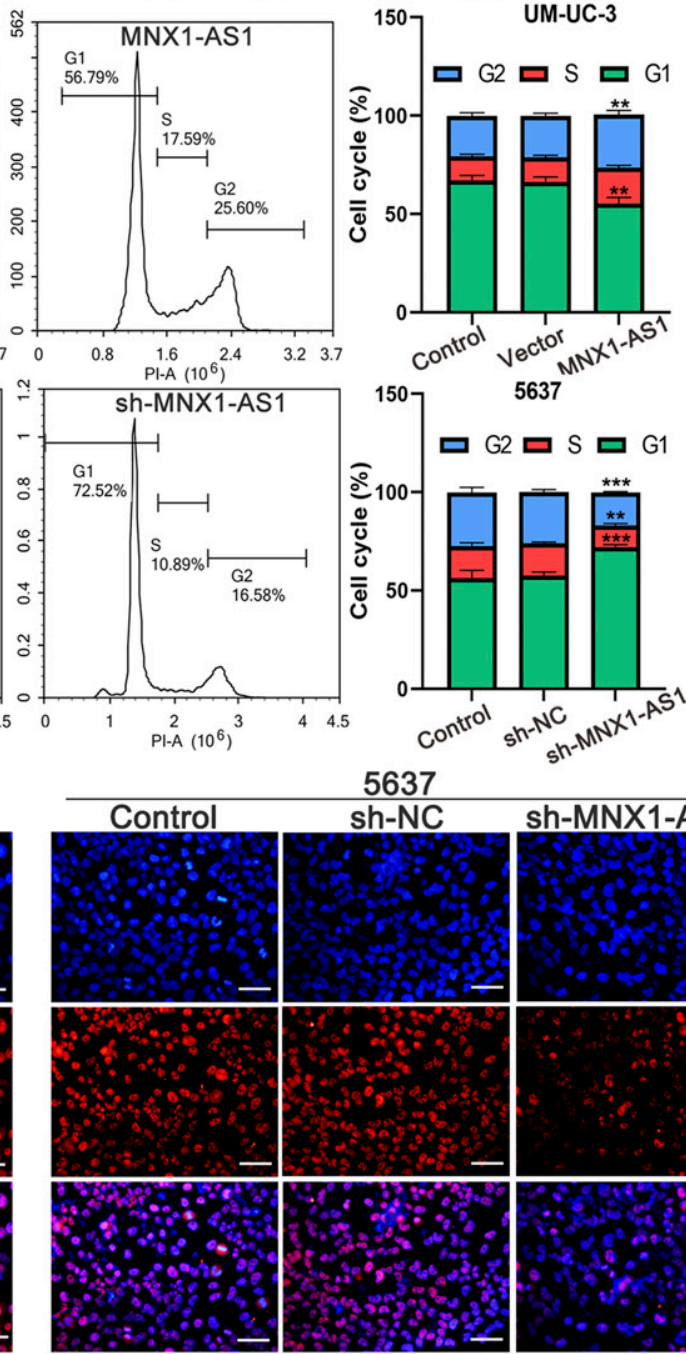

5637

sh-NC sh-MNX1-AS1
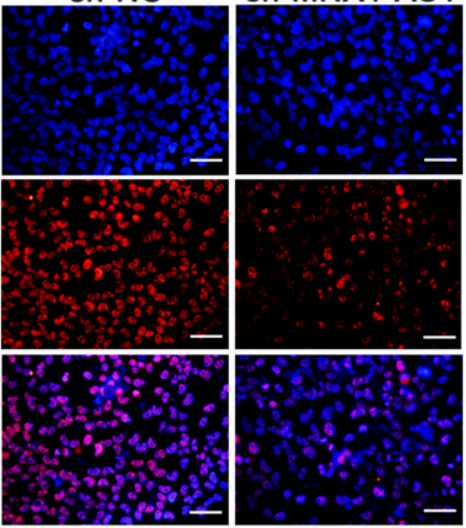

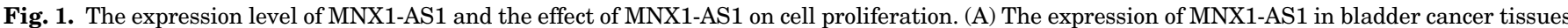

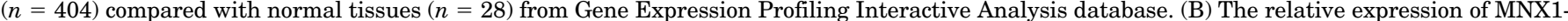

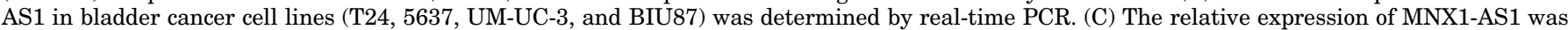

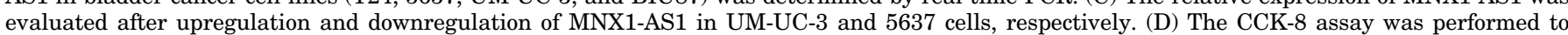

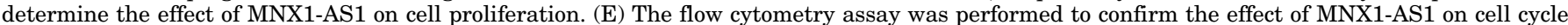


as the competing endogenous RNA by modulating miR-218-5p in human cancer, suggesting that MNX1-AS1 contains complementary binding sites for miR-218-5p (Ye et al., 2019). In addition, the antitumor effect of miR-218-5p has been widely reported (Cheng et al., 2015; Li et al., 2017; Xu et al., 2018; Wu et al., 2019; Xuan et al., 2019). miR-218-5p can restrain cell proliferation, migration, and invasion of bladder cancer cells and promote sensitivity to cisplatin in bladder cancer cells (Cheng et al., 2015; Li et al., 2017). Therefore, we speculated that MNX1-AS1 could affect the proliferation, migration, and invasion of bladder cancer cells via miR-218-5p.

In the present study, we found that the expression levels of MNX1-AS1 were higher in bladder tumor tissues compared with normal bladder tissues. We also found that MNX1AS1 promoted cell proliferation, migration, invasion, and epithelial-mesenchymal transition (EMT) of bladder cancer cells. Moreover, MNX1-AS1 could function as a sponge of miR218-5p, interfere with the expression of miR-218-5p-target gene RAB1A, and affect the development of bladder cancer. Therefore, we demonstrated that MNX1-AS1 plays a key role in bladder cancer and may serve as a therapeutic target and potential biomarker in bladder cancer.

\section{Materials and Methods}

Cell Culture. Bladder cancer cells (T24, 5637, UM-UC-3, and BIU-3) were purchased from Zhongqiao xinzhou Biologic Technology Co., Ltd. (Shanghai, China). T24, 5637, and BIU-3 were cultured in Roswell Park Memorial Institute 1640 medium (Gibco) supplemented with 10\% FBS (Biologic Industries, Israel). UM-UC-3 was cultured in minimum Eagle's medium (MilliporeSigma) containing $10 \%$ fetal bovine serum. All cell lines were cultured in an atmosphere at $37^{\circ} \mathrm{C}$ with $5 \% \mathrm{CO}_{2}$.

Cell Transfection. Negative control shRNA (sh-NC) and shRNA targeting MNX1-AS1 (sh-MNX1-AS1) were constructed by Wanleibio (Shenyang, China). NC mimic/inhibitor, miR-218-5p mimic/inhibitor, NC siRNA, and RAB1A siRNA (si-RAB1A) were obtained from JTS scientific (Guangzhou, China). The bladder cancer cells were transfected with $20 \mu \mathrm{g} / \mathrm{ml}$ plasmid, $1 \mu \mathrm{M}$ shRNA, or $1 \mu \mathrm{M}$ mimic/inhibitor using Lipofectamine 2000 transfection reagent (Invitrogen). The 5637 cells were cotransfected with $0.5 \mu \mathrm{M}$ shRNA and $0.5 \mu \mathrm{M} \mathrm{NC} / \mathrm{miR}-218$ $5 p$ inhibitor using Lipofectamine 2000. UM-UC-3 was cotransfected with $0.5 \mu \mathrm{M}$ siRNA and $10 \mu \mathrm{g} / \mathrm{ml}$ plasmid using Lipofectamine 2000 . Then these bladder cancer cells were collected for other experiments after transfection for 48 hours.

RNA Extraction and Real-Time Polymerase Chain Reaction. Total RNA was extracted from bladder cancer cells using the TRIpure reagent (BioTeke, China). Then cDNA was synthesized by super moloney murine leukemia virus reverse transcriptase (BioTeke). The real-time polymerase chain reaction (PCR) was carried out with $2 \times$ Power Taq PCR MasterMix (BioTeke). The primers for real-time PCR were as follows: MNX1-AS1 forward primer: 5'-CCC GCATTTTCAGATTCAC-3', reverse: 5'-GCTCTCAGCCTCGCCATA$3^{\prime}$; RAB1A forward primer: $5^{\prime}$-GCCTTCTTCTTAGGTTTG-3', reverse: $5^{\prime}$-TCTGTAATAACTGGAGGTG-3'; $\beta$-actin forward primer: $5^{\prime}$-GGC ACCCAGCACAATGAA-3', reverse: 5 '-TAGAAGCATTTGCGGTGG3'; miR-218-5p forward primer: 5'-TTGTGCTTGATCTAACCATGT3', reverse: 5'-GCAGGGTCCGAGGTATTC-3'; and U19 forward primer: 5'-TGGAGTTGATCCTAGTCTGG-3', reverse: 5'-GCAGGG
TCCGAGGTATTC-3'. Real-time PCR data were collected and analyzed using Exicycler 96 Software (Bioneer, Korea). Relative expression levels were calculated using the $2^{-\Delta \Delta \mathrm{Ct}}$ method.

Cell Counting Kit-8 Assay. Cell proliferation of bladder cancer cells was detected using the Cell Counting Kit-8 assay (CCK-8; MilliporeSigma). The 5637 and UM-UC-3 cells were seeded in different 96 -well plates at a density of $3 \times 10^{3}$ cells/well. Each group was prepared with five repeated wells. Culture plates were removed after transfection for $0,24,48,72$, and 96 hours, respectively. Next, $10 \mu \mathrm{l} \mathrm{CCK}-8$ was added to each well at $37^{\circ} \mathrm{C}$ for 2 hours. The optical density values were measured using ELX-800 Microplate Reader (BIOTEK) at $450 \mathrm{~nm}$.

Flow Cytometry Analysis. For cell cycle analysis, we used the Cell Cycle Kit (Beyotime, China). After transfection, the cells were harvested and fixed with $1 \mathrm{ml} 70 \%$ absolute ethanol at $4^{\circ} \mathrm{C}$ overnight. Then, cells were washed with PBS twice and centrifuged at $1000 \mathrm{~g}$ for 5 minutes to collect these cells. Next, these cells were incubated with $25 \mu \mathrm{l}$ of propidium iodide liquid and $10 \mu \mathrm{l}$ Rase $\mathrm{A}$ at $37^{\circ} \mathrm{C}$ for 30 minutes and measured by NovoCyte Flow Cytometer (ACEA Biosciences, China).

Wound-Healing Assay. Twenty-four hours after transfection, the monolayer cell was scratched with the $200 \mu \mathrm{l}$ pipette tip. Meanwhile, the medium was replaced with a serum-free medium. These cells were treated with a medium containing $1 \mathrm{mM}$ mitomycin (MilliporeSigma) to inhibit cell division. Each scratch wound was recorded with a microscope at the same position at 0 and 24 hours, respectively. The experiments were conducted in triplicate.

Transwell Assay. The capacity of cell invasion was assessed by Transwell assay. First, the transwell chamber was covered with Matrigel (BD). The bladder cancer cells were seeded on the upper chamber and incubated with serum-free culture medium. Meanwhile, $800 \mu \mathrm{l}$ medium was added in the lower chamber, with $30 \%$ serum acting as chemoattractant. After 24-hour culture, the bladder cancer cells in the upper chamber were washed twice with PBS. The lower cells were fixed with $4 \%$ paraformaldehyde for 15 minutes and subsequently stained with $0.4 \%$ crystal violet stain (Amresco) for 5 minutes. Five randomly microscopic fields were selected for analysis.

Immunofluorescence. Cells were seeded on the coverslips and cultured for proper cell density. After fixing by paraformaldehyde, the cells were blocked with FBS. Then, the cells were incubated with E-cadherin mouse antibody (dilution of 1:200; Cell Signaling Technology) or Ki-67 rabbit antibody (dilution of 1:200; Abcam, UK) overnight at $4^{\circ} \mathrm{C}$, respectively. The samples were then washed with PBST for 3 minutes and incubated with fluorescein Cy3-conjugated secondary antibodies (Beyotime). The incubation time of each secondary antibody was 60 minutes. 4,6-diamidino-2-phenylindole (Beyotime) was used for counterstaining. All antibodies were diluted with PBS. Images were captured by a fluorescence microscope (Olympus, Japan).

Dual-Luciferase Reporter Assay. We generated the RAB1A wild-type luciferase plasmid containing potential miR-218-5p binding sites (RAB1A-wt) and mutated versions of these sites (RAB1A-mut). RAB1A-wt/-mut was cotransfected with miR-218-5p mimic/inhibitor into 293T cells using Lipofectamine 2000 reagent (Invitrogen). Forty-eight hours after transfection, renilla and firefly luciferase activities were tested by the Dual-Luciferase Reporter assay system (Promega).

Western Blot Analysis. Bladder cancer tissues and cells were lysed in radio immunoprecipitation assay lysis buffer (Beyotime), and the protein concentrations were assayed by the bicinchoninic acid protein assay kit (Beyotime). Proteins were separated by sodium dodecyl sulfate-polyacrylamide gel electrophoresis (Beyotime) and

distribution in bladder cancer cells. (F) Immunofluorescence staining was performed to detect the effect of MNX1-AS1 on the expression of Ki-67 in bladder cancer cells. Scale bar, $50 \mu \mathrm{m}$. Each experiment was independently repeated at least three times. Data are shown as mean \pm S.D.; $* * P<0.01$; $* * * P<0.001$. G. gap; S, synthesis. 
A A
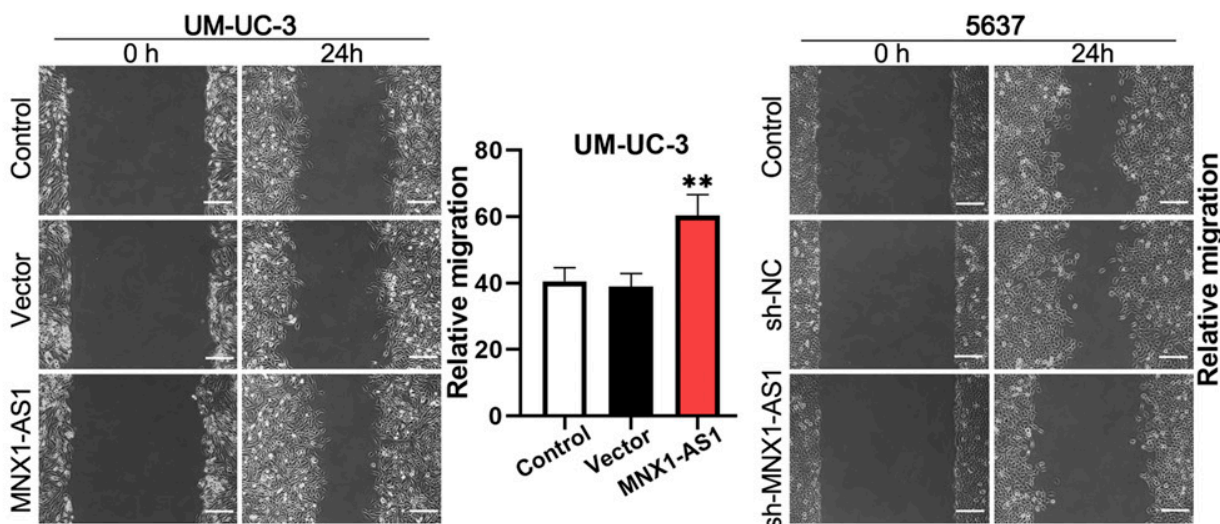

B

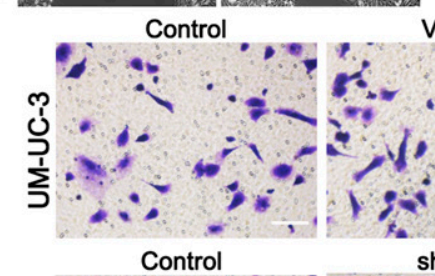

Vectror

MNX1-AS1
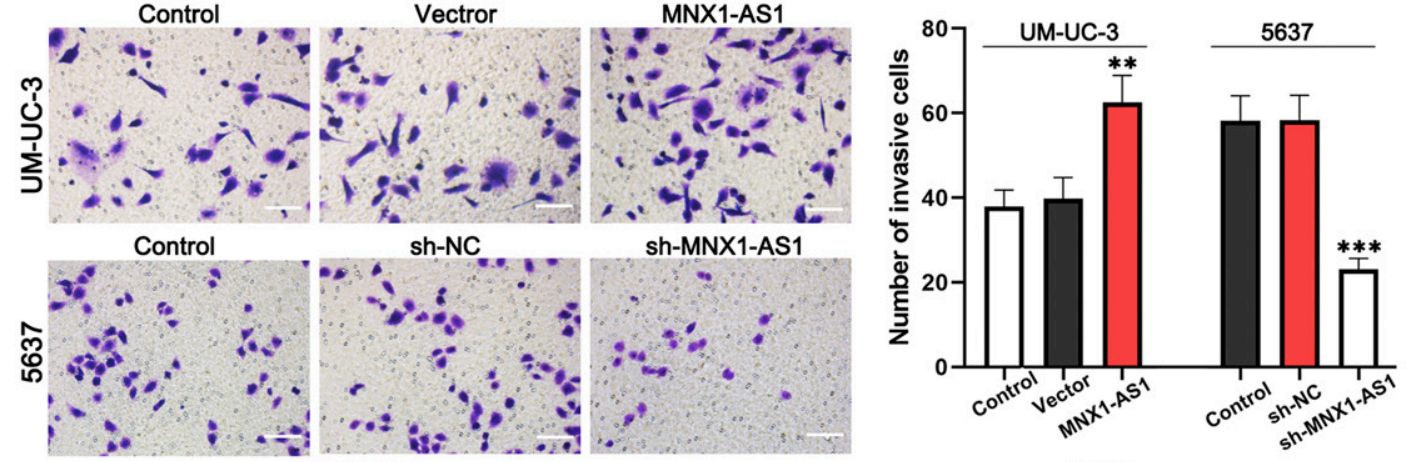

C

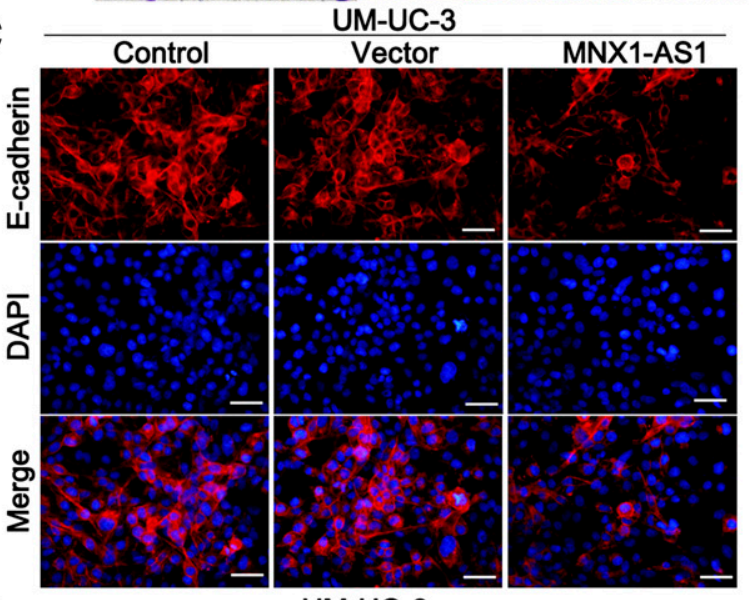

D

UM-UC-3
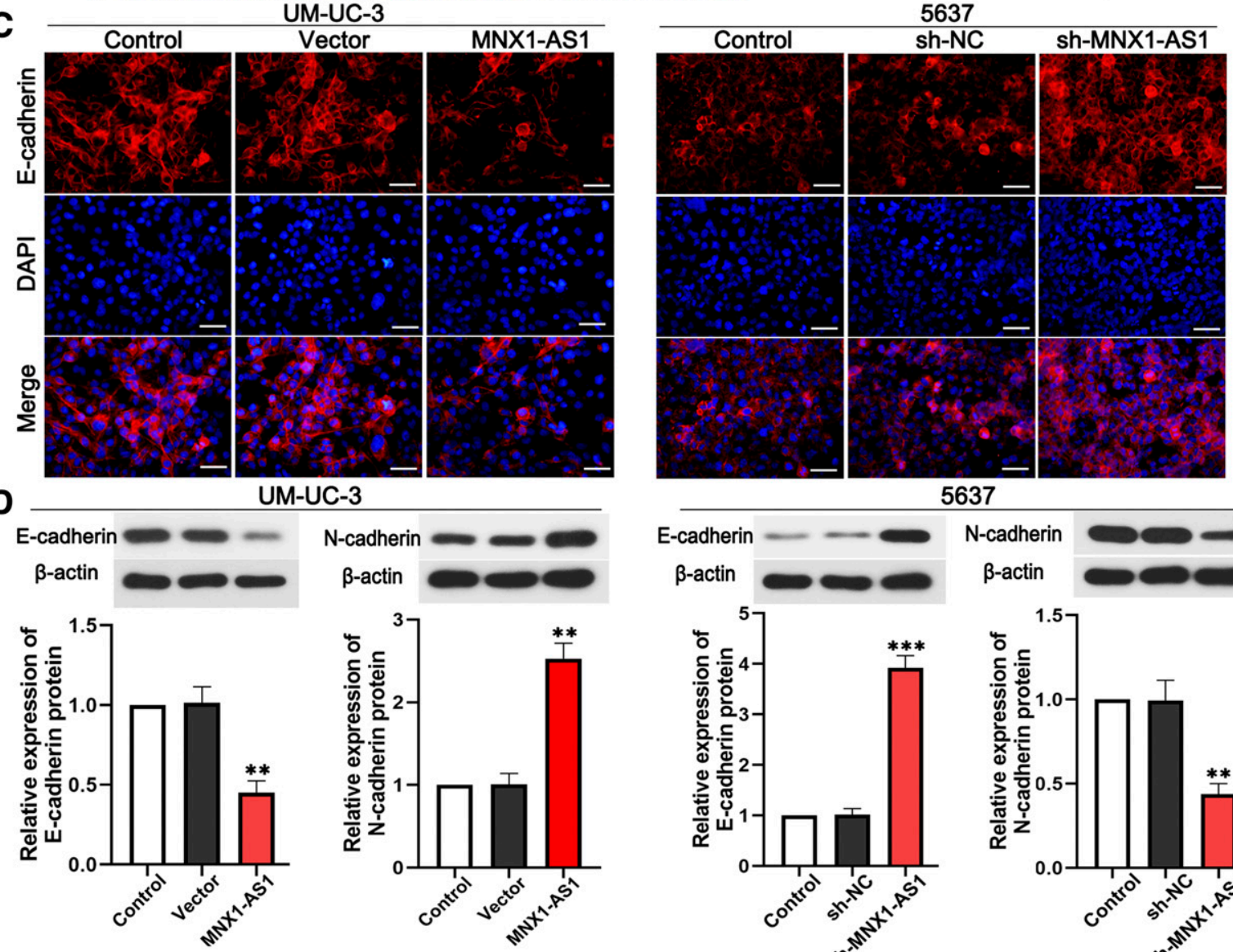

5637

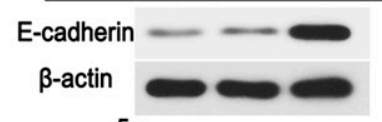

$\mathrm{N}$-cadherin
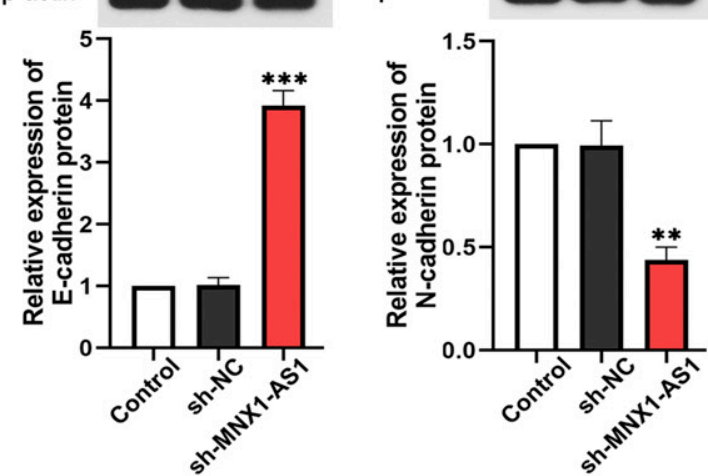

Fig. 2. MNX1-AS1 influenced the cell migration, invasion, and EMT phenotypes of bladder cancer cells. The UM-UC-3 cells were transfected with vector or MNX1-AS1, and 5637 cells were transfected with sh-NC or sh-MNX1-AS1. (A) Wound-healing assay was performed to detect the effect of MNX1-AS1 on cell migration. Scale bar, $200 \mu \mathrm{m}$. (B) The effect of MNX1-AS1 on cell invasion was detected by transwell assay. Scale bar, $100 \mu \mathrm{m}$. (C) Immunofluorescence staining was performed to examine the effect of MNX1-AS1 on the expression of E-cadherin. Scale bar, $50 \mu \mathrm{m}$. (D) Western blot was performed to test the E-cadherin and N-cadherin protein expression. Each experiment was independently repeated at least three times. Data are shown as mean \pm S.D.; $* * P<0.01 ; * * * P<0.001$. 
A

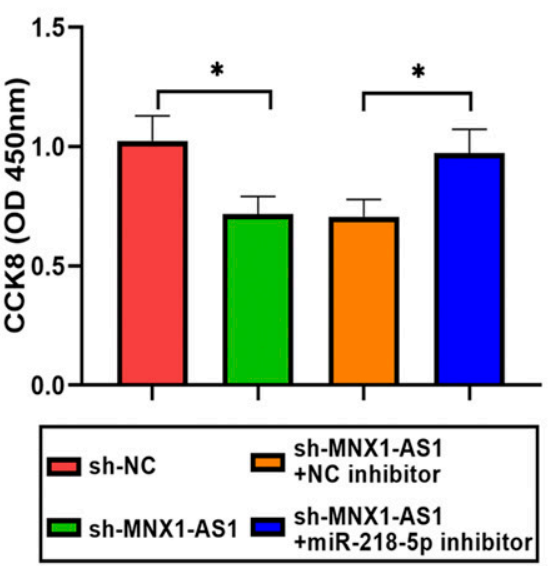

B

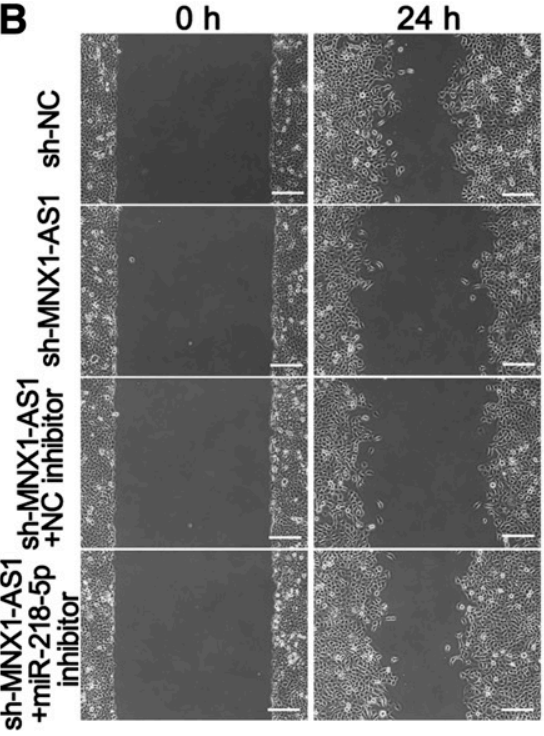

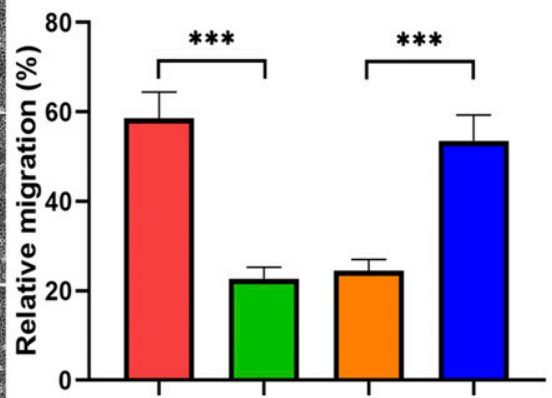

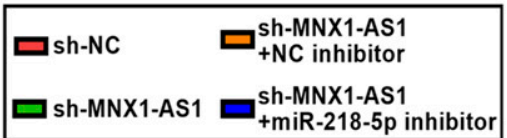

C

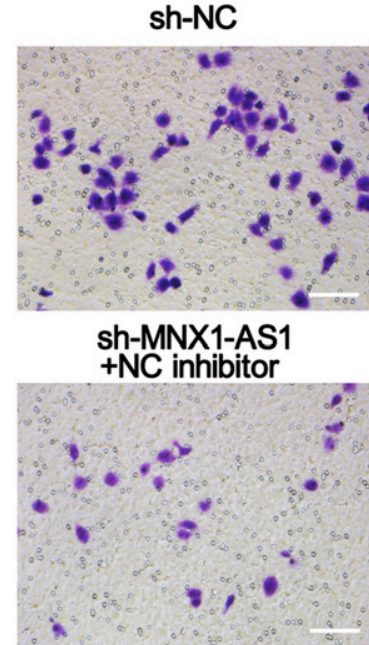

sh-MNX1-AS1

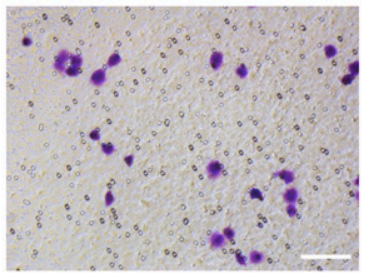

sh-MNX1-AS1 + miR-218-5p inhibitor

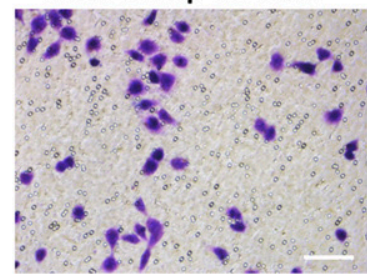

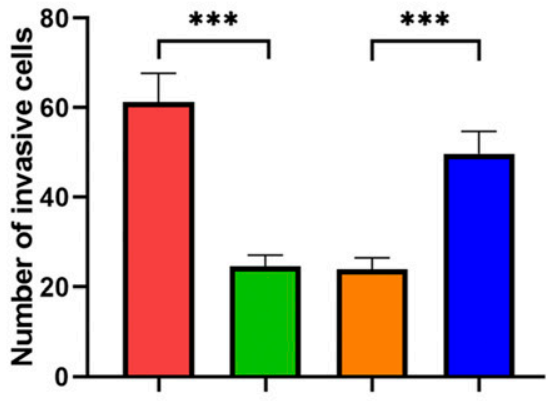

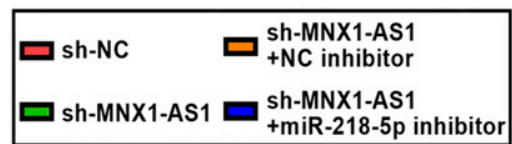

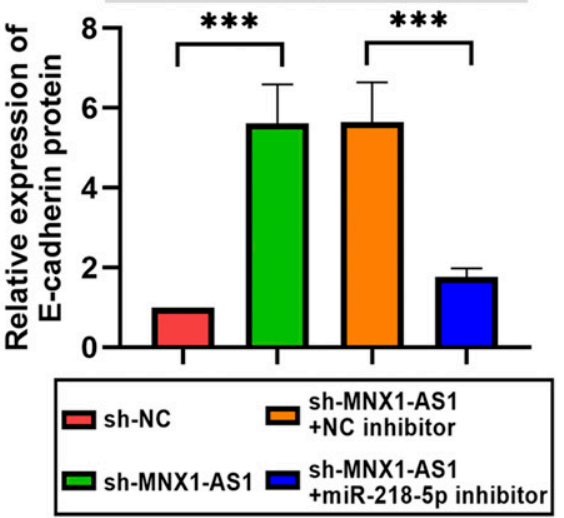

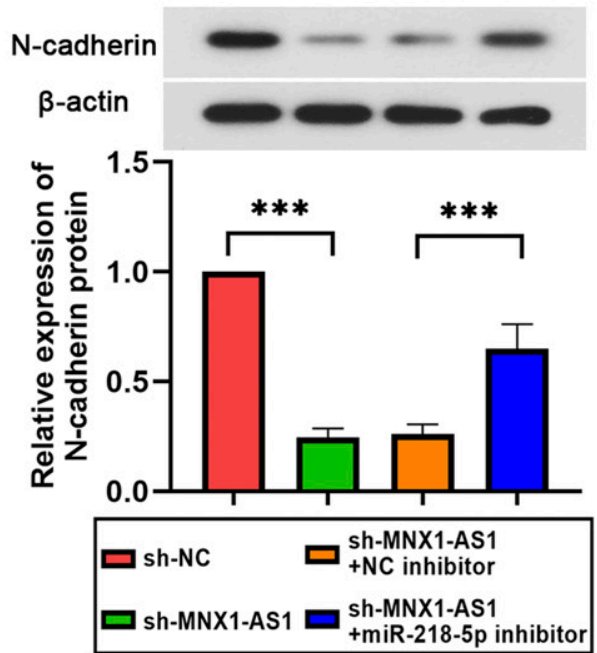

Fig. 3. MNX1-AS1 affected the cell proliferation, migration, invasion, and EMT of bladder cancer cells via miR-218-5p. Sh-MNX1-AS1 and miR-218$5 \mathrm{p} / \mathrm{NC}$ inhibitor were cotransfected in 5637 cells. (A) Cell proliferation was analyzed by CCK-8 assay. (B) Cell migration ability was confirmed by wound-healing assay. Scale bar, $200 \mu \mathrm{m}$. (C) Transwell assay was carried out to measure the invasion. Scale bar, $100 \mu \mathrm{m}$. (D) The protein levels of E-cadherin and N-cadherin were detected by Western blot. Each experiment was independently repeated at least three times. Data are shown as mean \pm S.D.; $* P<0.05 ; * * * P<0.001$. 
A

RAB1A-wt: 5'-AUUUUGUAUCAAACAGCACAA-3' miR-218-5p : 3'-UGUACCAAUCUAGUUCGUGUU-5' RAB1A-mut : 5'-AUUUUGUAUCAAACTCGTGTA-3'

B

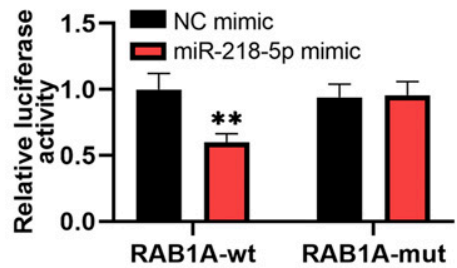

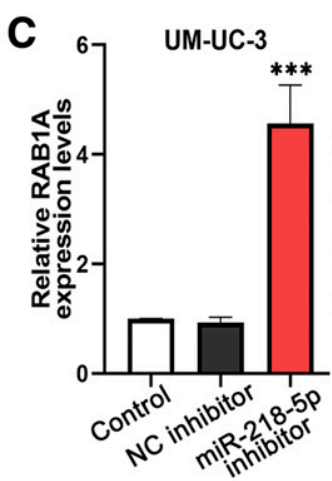

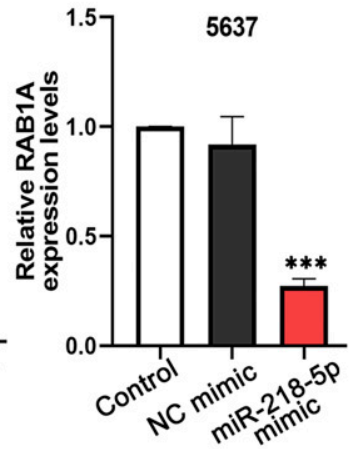

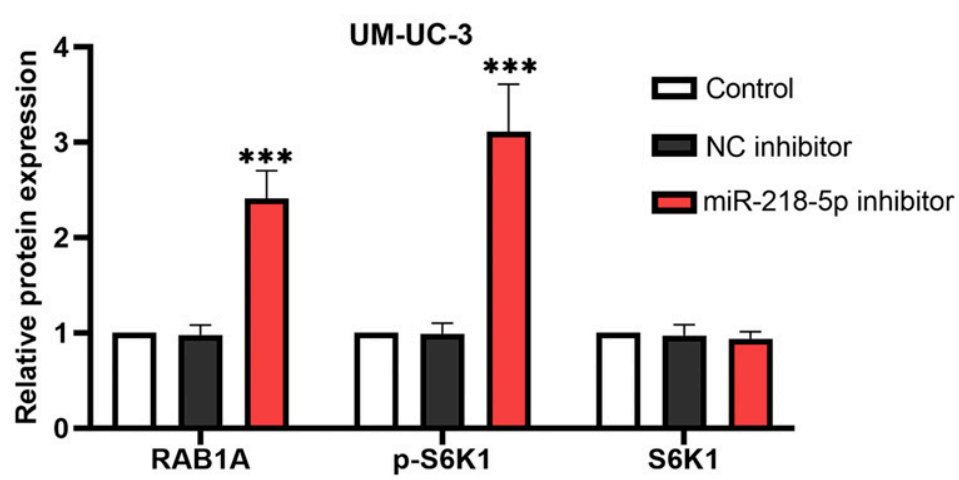

E

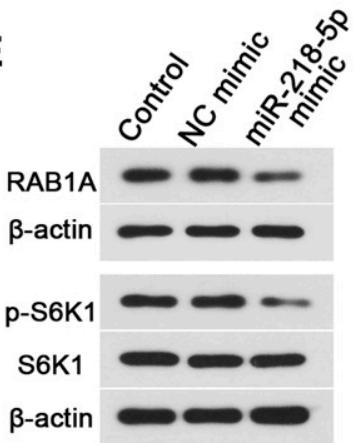

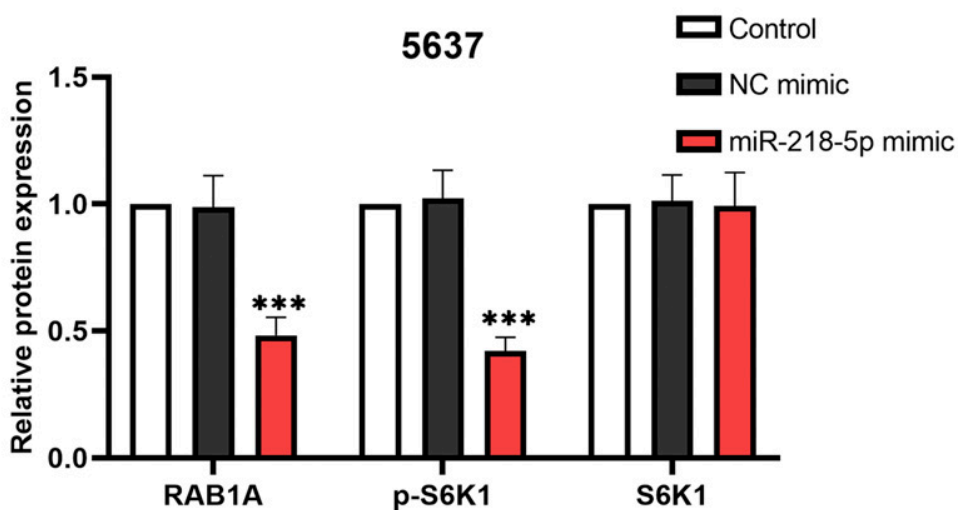

Fig. 4. The effect of miR-218-5p on RAB1A and its related proteins. (A) Schematic of forecasted wild type or mutated miR-218-5p binding sequences in RAB1A. (B) Luciferase activity of $293 \mathrm{~T}$ cells cotransfected with miR-218-5p mimics or NC mimics and luciferase reporters containing RAB1A-wt or RAB1A-mut transcript were determined by dual-luciferase reporter assays. (C) After upregulation or downregulation of miR-218-5p in bladder cancer cells, the relative expression of RAB1A was determined by real-time PCR. (D) The protein levels of RAB1A, p-S6K1, and S6K1 were detected by Western blot in UM-UC-3 cells transfected with miR-218-5p inhibitor and NC inhibitor. (E) The protein levels of RAB1A, p-S6K1, and S6K1 were detected by Western blot in 5637 cells transfected with miR-218-5p mimic and NC mimic. Each experiment was independently repeated at least three times. Data are shown as mean \pm S.D.; $* * P<0.01 ; * * * P<0.001$.

transferred onto polyvinylidenedi fluoride membrane (Thermo Fisher Scientific). After blocking with $5 \%$ bovine serum albumin (Biosharp, China) in PBST, these membranes were incubated with $\beta$-actin mouse antibody (dilution of 1:2000; PProteintech, China), RAB1A rabbit antibody (dilution of 1:1000; PProteintech), phosphorylated S6 kinase 1 (p-S6K1) rabbit antibody (dilution of 1:500; Cell Signaling Technology), S6K1 (dilution of 1:1000; Cell Signaling Technology), E-cadherin mouse antibody (dilution of 1:500; Cell Signaling Technology), and N-cadherin rabbit antibody (dilution of 1:300; Cell Signaling Technology) at $4^{\circ} \mathrm{C}$ for overnight. The incubation time of different primary antibodies was the same. The membranes were then washed with PBST and incubated with goat anti-mouse IgG secondary antibody (dilution of 1:10,000; ProteintechProteintech) or goat anti-rabbit IgG secondary antibody (dilution of $1: 10,000$; PProteintech) at $37^{\circ} \mathrm{C}$. The incubation time of each secondary antibody was 40 minutes. All antibodies were diluted with 5\% BSA. Antigen-antibody reaction was observed by electrogenerated chemiluminescence assay (7 Sea Biotech, China).

Immunohistochemistry. Paraffin-embedded sections that were $5-\mu \mathrm{m}$ thick were used for immunohistochemistry (IHC) staining. Samples were incubated with E-cadherin mouse antibody (dilution of 1:200; Cell Signaling Technology) and Ki-67 rabbit antibody (dilution of 1:200; Abcam) overnight at $4^{\circ} \mathrm{C}$. After washing with PBS, samples were incubated with horseradish peroxidase-conjugated secondary antibody (Thermo Fisher Scientific) for 60 minutes at $37^{\circ} \mathrm{C}$. All antibodies were diluted with PBS. Then, these samples were stained with diaminobenzidine (Solarbio, China) and counterstained with hematoxylin (Solarbio).

Tumor Xenograft Model. Two-month male nude mice (18-20 g) were purchased from Beijing HFK Bioscience Co. Ltd. (Beijing, China). Environmental conditions were kept constant (lights on at 
A

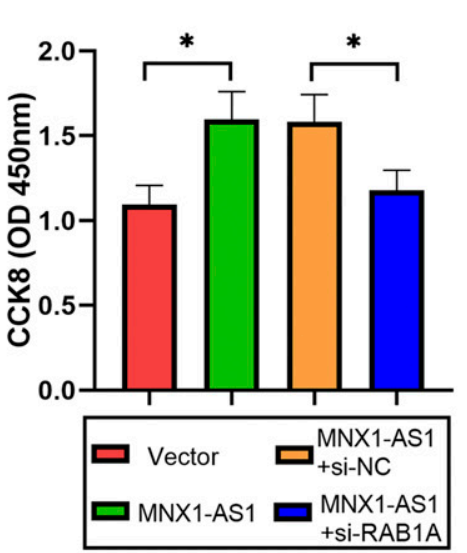

B

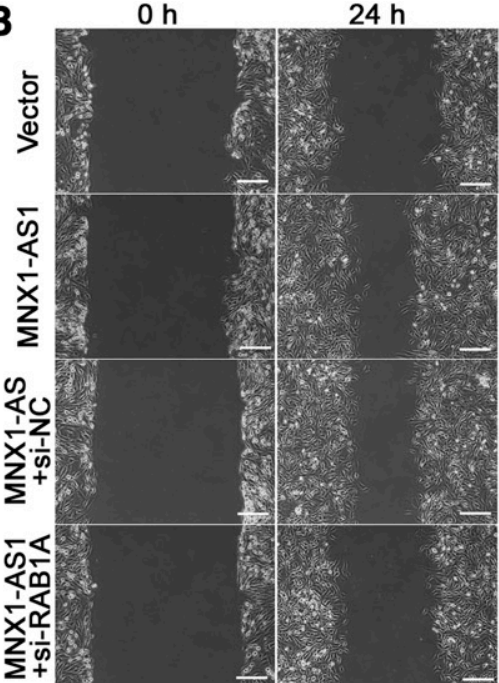
MNX1-AS1

C
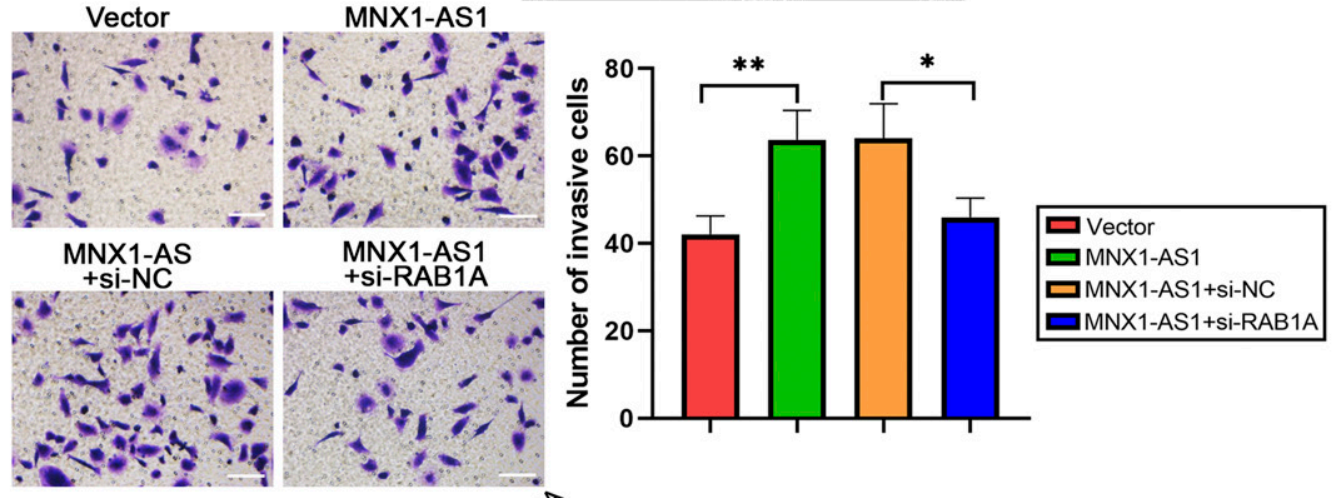

D
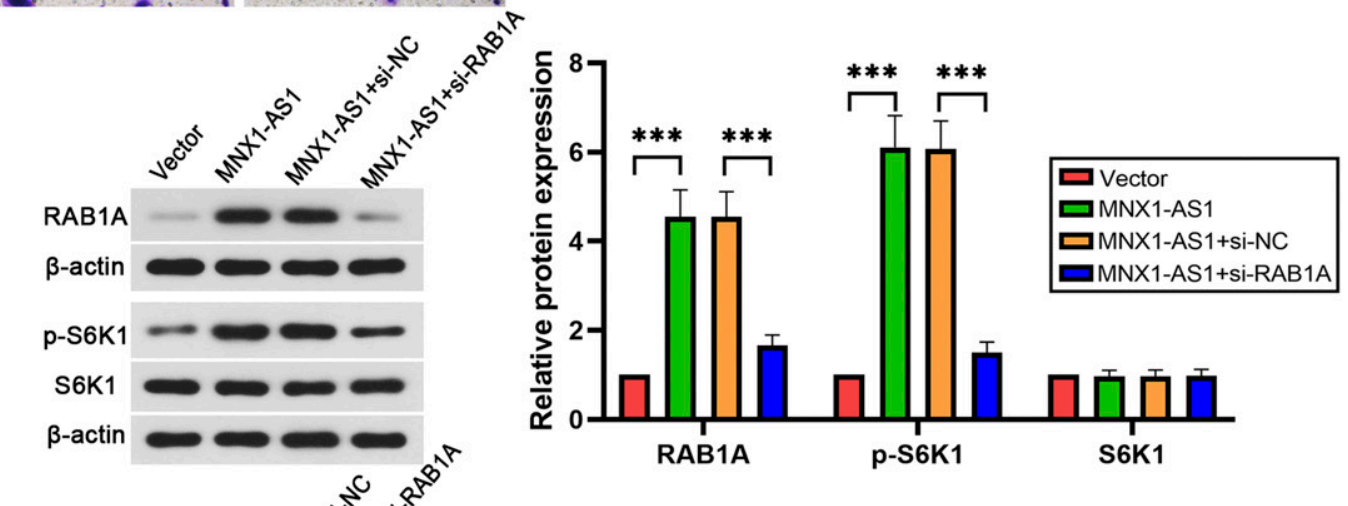

$\mathbf{E}$

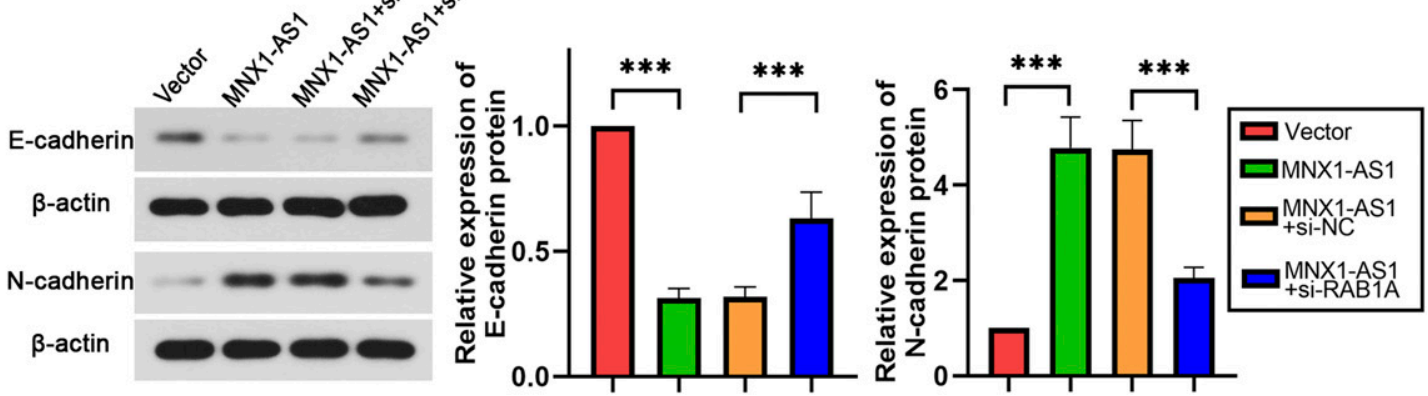

Fig. 5. MNX1-AS1 affected the cell proliferation, migration, invasion, and EMT of bladder cancer cells via RAB1A. MNX1-AS1 and RAB1A/NC siRNA were cotransfected in UM-UC-3 cells. (A) Cell proliferation was analyzed by CCK-8 assay. (B) Wound-healing assay was carried out to confirm cell migration ability. Scale bar, $200 \mu \mathrm{m}$. (C) The invasion was measured by transwell assay. Scale bar, $100 \mu \mathrm{m}$. (D) The protein levels of RAB1A, p-S6K1, and S6K1 were detected by Western blot in UM-UC-3 cells. (E) The expression of N-cadherin and E-cadherin was detected by Western blot after transfection. Each experiment was independently repeated at least three times. Data are shown as mean \pm S.D.; ${ }^{*} P<0.05 ; * * P<0.01 ; * * * P<0.001$. 


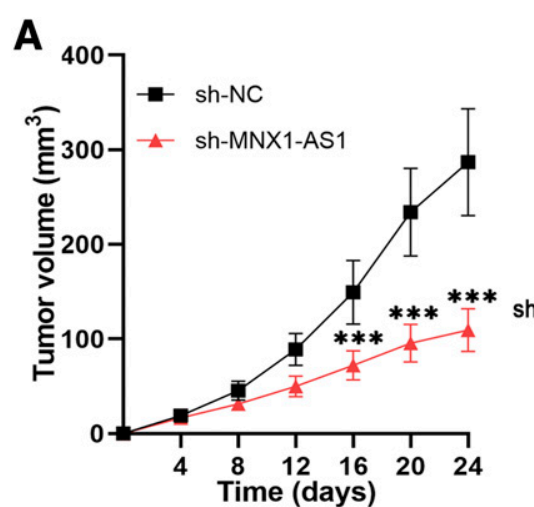

C
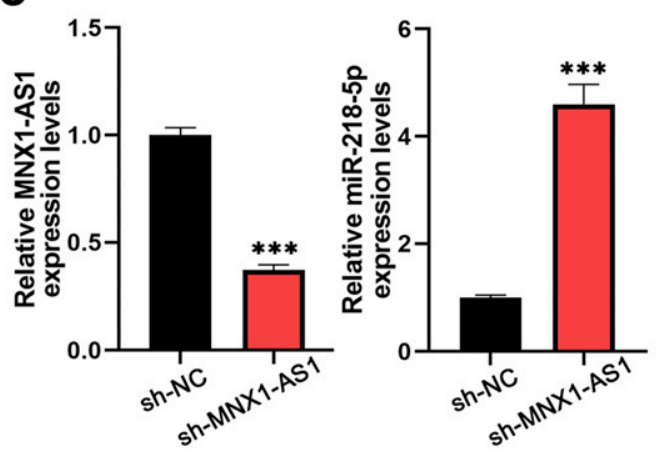

$\mathbf{E}$

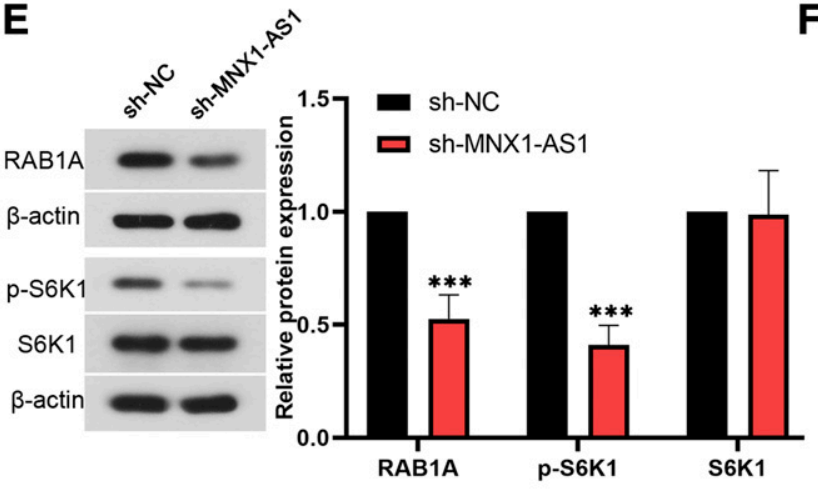

B

$\mathbf{F}$
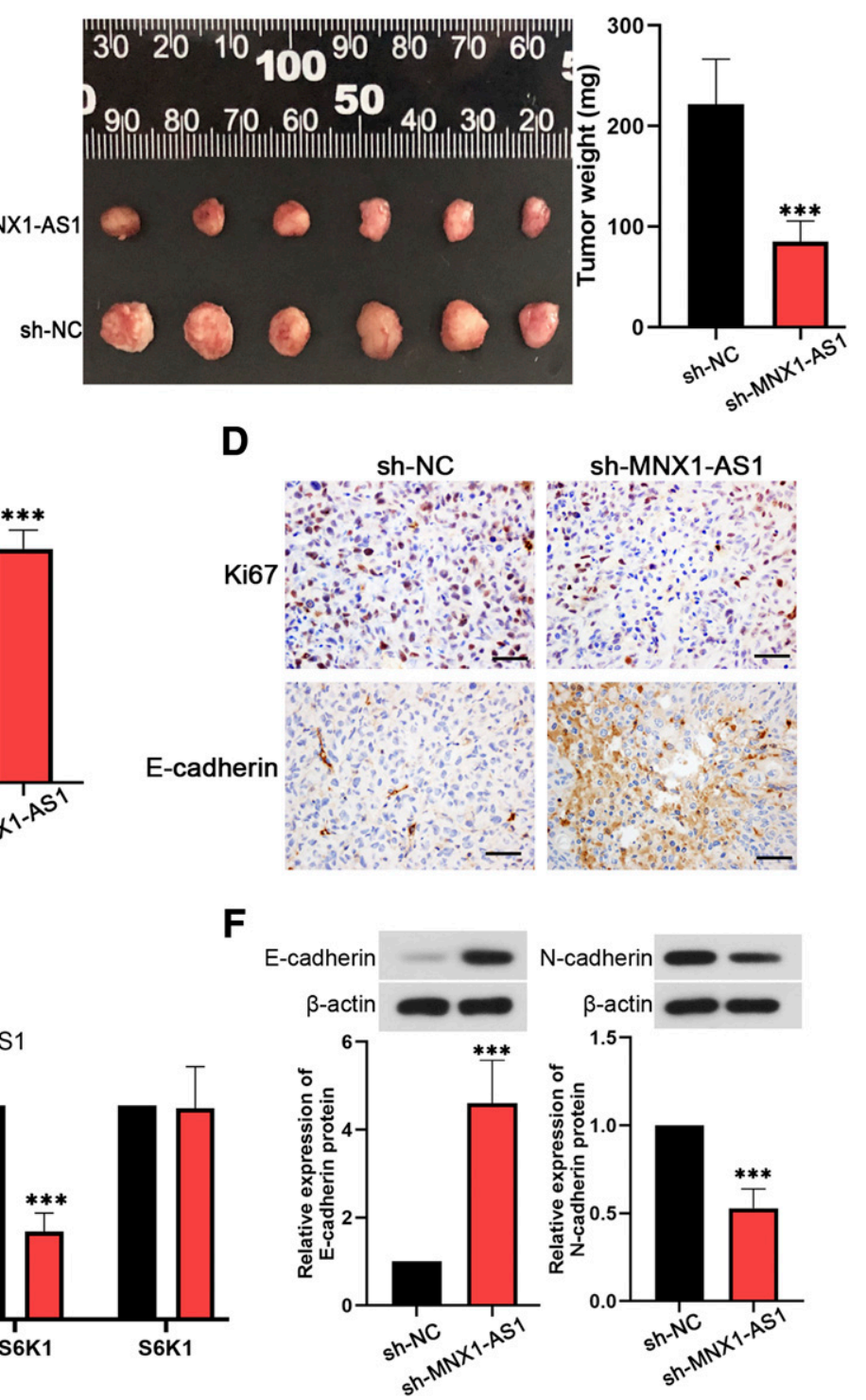

Fig. 6. MNX1-AS1 influenced bladder cancer growth and EMT in vivo. Nude mice were injected with sh-MNX1-AS1 and sh-NC by tail intravenous injection ( $n=6$ per group). (A) The volume of tumors was measured every 4 days for 24 days. (B) The volume and weight of the tumors were recorded on the 24th day. (C) The relative expression of MNX1-AS1 and miR-218-5p in tumor tissues was evaluated by real-time PCR. (D) The levels of Ki-67 and E-cadherin expression in tissues were determined by IHC. Scale bar, $50 \mu \mathrm{m}$. (E) The protein levels of RAB1A, p-S6K1, and S6K1 were detected by Western blot. (F) The protein expression of EMT markers (E-cadherin and N-cadherin) in cancer tissues was determined by Western blot. Each experiment was independently repeated at least three times. Data are shown as mean \pm S.D.; ***P $<0.001$.

06:00 hours and off at 18:00 hours; humidity, 45\%-55\%; temperature, $\left.22 \pm 1^{\circ} \mathrm{C}\right)$. Nude mice were given ad libitum chow and water for 1 week. Then, 5637 were injected subcutaneously into the right axilla of nude mice. When bladder tumors were visible to the naked eye, nude mice were injected with sh-NC and sh-MNX1-AS1 by tail intravenous injection every 3 days, and tumor volumes were recorded every 4 days. The mice were sacrificed after 24 days, and tumor xenografts were harvested, weighed, and subjected to real-time PCR, Western blot, and immunohistochemical examination. All animal experiments were performed in accordance with the Guide for the Care and Use of Laboratory Animals, and this research was approved by the Animal Care and Use Committee of the First Affiliated Hospital of Zhengzhou University.

Statistical Analysis. GraphPad Prism 8 was used to analyze experimental data. A $t$ test was used to analyze the data differences between the two groups. Multiple groups of data were compared by one-way ANOVA. Data were presented as mean \pm S.D. from three independent experiments. The value of $P<0.05$ was considered statistically significant.

\section{Results}

The Effect of MNX1-AS1 on Cell Proliferation of Bladder Cancer Cells. The expression levels of MNX1-AS1 in normal and tumor patients were analyzed by Gene Expression Profiling Interactive Analysis (http://gepia. cancer-pku.cn/). The results showed that MNX1-AS1 was markedly upregulated in bladder tumor tissues compared with normal bladder tissues (Fig. 1A). Then, we investigated the expression of MNX1-AS1 in bladder cancer cells (T24, 5637, UM-UC-3, and BIU-87). The highest level was detected 
in 5637 cells, and the lowest level was detected in UM-UC-3 cells (Fig. 1B). Further experiments indicated that overexpression of MNX1-AS1 could upregulate the expression level of MNX1-AS1 in UM-UC-3 cells, whereas the MNX1AS1-specific shRNA could downregulate MNX1-AS1 levels in 5637 cells (Fig. 1C). Next, CCK-8 assay demonstrated that overexpression of MNX1-AS1 promoted cell proliferation of bladder cancer cells, and sh-MNX1-AS1 dramatically suppressed the cell proliferation compared with the negative control (Fig. 1D). The flow cytometry results shown in Fig. 1E indicated that the percentage of cells in the Gap 1 phase was decreased $(66.4 \% \pm 2.5 \%$ to $55.3 \% \pm 3.1 \%)$ and Gap 2 phase was increased $(21.2 \% \pm 1.3 \%$ to $25 \% \pm 1.8 \%)$ through promoting MNX1-AS1 expression in UM-UC-3 cells, whereas downregulated MNX1-AS1 expression in 5637 cells improved the percentage of cells in the Gap 1 phase $(57.6 \% \pm 1.8 \%$ to $72.1 \% \pm 1.16 \%)$ and reduced the percentage of cells in Synthesis phase $(16.5 \% \pm 0.5 \%$ to $10.9 \% \pm 1.0 \%)$ and Gap 2 phase $(25.8 \% \pm 1.3 \%$ to $16.8 \% \pm 0.3 \%)$. Additionally, Ki-67 immunostaining was stronger in UM-UC-3 cells transfected with the overexpression vector of MNX1-AS1 and weaker in 5637 cells transfected with sh-MNX1-AS1 (Fig. 1F). These results revealed that MNX1-AS1 promoted the proliferation of bladder cancer cells.

The Effect of MIX1-AS1 on Migration, Invasion, and EMT of Bladder Cancer Cells. Tumor metastasis is the main cause of bladder cancer treatment failure. EMT promotes the invasion and metastasis of bladder cancer (Yun and Kim, 2013; Yang et al., 2016). The wound-healing assay was performed to measure the migration ability of bladder cancer cells (Fig. 2A). The migration rate of UM-UC-3 cells with MNX1-AS1 overexpression was higher than that of UM-UC-3 cells transfected with vector $(60.3 \% \pm 6.3 \%$ vs. $38.9 \% \pm 3.9 \%)$. The migration rate of 5637 cells with sh-MNX1-AS1 was less than that of 5637 cells with sh-NC $(24.8 \% \pm 3.0 \%$ vs. $60.5 \% \pm$ $6.4 \%$ ) (Fig. 2A). In addition, transwell assay was performed to measure the invasion ability of bladder cancer cells (Fig. 2B). The invasion cell numbers of UM-UC-3 with MNX1-AS1 overexpression were more than that of UM-UC-3 transfected with vector $(62.5 \pm 6.4$ vs. $39.8 \pm 5.0)$. The 5637 cells with shMNX1-AS1 showed weaker invasion ability compared with 5637 cells with sh-NC $(23.1 \pm 2.6$ vs. $58.3 \pm 5.9)$ (Fig. $2 B)$. These results revealed that MNX1-AS1 overexpression enhanced the migration and invasion of UM-UC-3 cells. Inhibited cell migration and invasion were observed in 5637 cells induced by silencing MNX1-AS1. The expression level of E-cadherin (a marker of EMT) was determined using immunofluorescence (Fig. 2C) and Western blot (Fig. 2D), and the expression level of N-cadherin (a marker of EMT) was determined by Western blot (Fig. 2D). MNX1-AS1 overexpression decreased E-cadherin expression and increased $\mathrm{N}$-cadherin expression in UM-UC-3 cells, whereas knockdown of MNX1AS1 had the opposite effects. These results revealed that MNX1-AS1 promoted the migration, invasion, and EMT of bladder cancer cells.

MNX1-AS1 Regulated Cell Proliferation, Migration, Invasion, and EMT of Bladder Cancer Cells via miR-218-5p. A recent study showed that MNX1-AS1 could bind to miR-218-5p (Ye et al., 2019). To determine whether MNX1-AS1 affected bladder cancer by modulating miR-218$5 \mathrm{p}, 5637$ cells were cotransfected with sh-MNX1-AS1 and NC/miR-218-5p inhibitor. Proliferation of 5637 cells was diminished after shRNA knockdown of MNX1-AS1, whereas cell proliferation was increased by downregulating miR-218$5 p$ expression (Fig. 3A). Besides, the migration rate of 5637 cells was reduced by sh-MNX1-AS1 $(22.7 \% \pm 2.6 \%)$ compared with sh-NC $(58.6 \% \pm 5.8 \%)$, whereas the migration rate could be increased by miR-218-5p inhibitor $(53.57 \% \pm 5.7 \%)$ (Fig. 3B). The invasion cell numbers of 5637cells were decreased by sh-MNX1-AS1 $(24.5 \pm 2.5)$ compared with sh-NC $(61.3 \pm 6.4)$, whereas invasion cell numbers were increased by miR-218-5p inhibitor (49.7 \pm 5.0 ) (Fig. 3C). Moreover, knockdown of MNX1-AS1 reduced the expression of E-cadherin protein and enhanced the expression of $\mathrm{N}$-cadherin protein, whereas this effect was reversed by miR218-5p inhibitor (Fig. 3D). Our results manifested that miR-218-5p inhibitor significantly reversed the inhibition of cell proliferation (Fig. 3A), migration (Fig. 3B), and invasion (Fig. 3C) induced by silencing MNX1-AS1. Therefore, MNX1-AS1 could promote the proliferation, migration, invasion, and EMT of bladder cancer cells by sponging miR-218-5p.

miR-218-5p Targeted RAB1A in Bladder Cancer Cells. The bioinformatic prediction (http://starbase.sysu. edu.cn) was performed to explore the target gene of miR218-5p. The prediction results showed that miR-218-5p might bind to RAB1A (Fig. 4A). There were reports that RAB1A could promote the progression of breast cancer, lung cancer, and tongue cancer (Shimada et al., 2005; Wang et al., 2016; Xu et al., 2017). However, its role in bladder cancer has not been reported.

Dual-luciferase reporter assay results demonstrated that the luciferase activity was significantly decreased in the cells cotransfected with miR-218-5p mimic and RAB1A-wt, whereas no significant difference was detected in the cells cotransfected with miR-218-5p mimic and RAB1A-mut (Fig. 4B). The result indicated that miR-218-5p could bind to RAB1A. Then, we performed real-time PCR to estimate the levels of RAB1A in bladder cancer cells transfected with miR218-5p inhibitor or mimic. The results showed that the levels of RAB1A were notably increased after being transfected with miR-218-5p inhibitor in UM-UC-3 cells, whereas the levels of RAB1A were markedly reduced in 5637 cells transfected with miR-218-5p mimic (Fig. 4C). There was evidence that RAB1A was closely related with p-S6K1 levels (Cheng et al., 2019). Therefore, we examined the protein expression levels of RAB1A and p-S6K1 after transfection of miR-218-5p inhibitor or miR-218-5p mimic in bladder cancer cells. miR-218-5p inhibitor could increase the expression levels of RAB1A and p-S6K1 in UM-UC-3 cells to about 2.4- and 3.1-fold, respectively (Fig. 4D). However, miR-218-5p mimic markedly decreased the expression levels of RAB1A and p-S6K1 in 5637 cells (Fig. 4E). These observations suggested that miR-218-5p could modulate the expression of RAB1A and the activation of S6K1.

MNX1-AS1 Regulated Cell Proliferation, Migration, Invasion, and EMT of Bladder Cancer Cells via RAB1A. We further tested whether MNX1-AS1 affected bladder cancer cells through RAB1A. CCK-8 assay indicated that cell viability was increased by MNX1-AS1, and this effect was abrogated by downregulating RAB1A (Fig. 5A).

The migration rate of UM-UC-3 cells was markedly promoted by MNX1-AS1 $(62.4 \% \pm 6.3 \%)$ compared with vector $(33.6 \% \pm 3.5 \%)$, whereas knockdown of RAB1A could reduce the migration rate $(44.3 \% \pm 4.6 \%)$ (Fig. 5B). The invasion cell 
numbers of UM-UC-3 cells were increased by MNX1-AS1 $(62.4 \pm 6.3)$, whereas this increase could be reversed by siRAB1A (44.3 \pm 4.6$)$ (Fig. 5C). In addition, the Western blot results proved that MNX1-AS1 overexpression enhanced the expression of RAB1A and p-S6K1 in UM-UC-3 cells (4.5- and 6.1-fold higher than that in vector group, respectively), whereas this effect was attenuated by si-RAB1A (Fig. 5D). Furthermore, si-RAB1A could reverse the increase in N-cadherin expression and decrease in E-cadherin expression induced by inhibition of MNX1-AS1 (Fig. 5E). Thus, these results proved that MNX1-AS1 could regulate proliferation, migration, invasion, and EMT of bladder cancer cells via RAB1A.

The Effect of MNX1-AS1 on Bladder Cancer Growth and EMT In Vivo. We further explored the role of MNX1AS1 in vivo by xenograft models. The bladder tumor growth and volumes of the sh-MNX1-AS1 group were markedly decreased, and this change was significant from day 16 (Fig. 6A). After 24 days, the mice were sacrificed, and tumor weight was determined. The tumor weight of sh-MNX1-AS1 group $(84.8 \pm 20.9 \mathrm{mg})$ was lighter than that of sh-NC group $(221.5 \pm 45.1 \mathrm{mg})$ (Fig. 6B). We found that knockdown of MNX1-AS1 inhibited MNX1-AS1 expression and promoted miR-218-5p expression in bladder tumor tissues (Fig. 6C). IHC results indicated that knockdown of MNX1-AS1 could markedly decrease the Ki-67 and increase the E-cadherin expression (Fig. 6D). Western blot results manifested that shMNX1-AS1 decreased RAB1A and p-S6K1 expression in vivo (Fig. 6E). In addition, Western blot results also showed that sh-MNX1-AS1 could affect the expression of E-cadherin and $\mathrm{N}$-cadherin in vivo (Fig. $6 \mathrm{~F}$ ). These observations suggested that sh-MNX1-AS1 could inhibit the development and metastasis of bladder cancer in vivo.

\section{Discussion}

Bladder cancer is a malignant tumor originating from the urothelium of the bladder (Wu et al., 2018). It is one of the most common malignant tumors in the urinary system (Wu et al., 2018). The incidence of bladder cancer is increasing, but the clinical treatment of bladder cancer has not changed dramatically (Ebrahimi et al., 2019). Surgery and postoperative complications seriously affect the quality of life of patients. As patients' requirements for quality of life improve, obtaining more precisely targeted bladder cancer therapies is an urgent problem to be solved. Although there are many studies on bladder cancer, the underlying mechanisms remain unclear. In our study, we demonstrated for the first time that MNX1-AS1 promotes bladder cancer development via the miR-218-5p/RAB1A axis.

LncRNAs are the key players in gene-regulation processes and are associated with carcinogenesis (Sun and Kraus, 2015; Hao et al., 2018). Previous studies showed that lncRNA MNX1-AS1 promoted the development and progression of various cancers (Lv et al., 2017b; Gao et al., 2019; Liu et al., 2019a,b; Ye et al., 2019). However, the effects of MNX1-AS1 on bladder cancer have not been reported. We found that the expression of MNX1-AS1 was obviously upregulated in bladder cancer patients. Our experiment results indicated that MNX1-AS1 could promote the proliferation of bladder cancer cells and affect cell cycle. Besides, cancer metastasis is a complex multistep process. The initial stage of metastatic progression is related to EMT, which is characterized by morphogenetic changes, the loss of cell polarity, and increased cell motility (Sleeman and Thiery, 2011; Spano et al., 2012). It has been reported that EMT can promote the invasion and metastasis of bladder cancer (Nieto et al., 2016; Brabletz et al., 2018). Therefore, elucidating the role of MNX1-AS1 in EMT could be crucial for understanding the regulatory mechanism of MNX1-AS1. We found that scratch wound was healed faster by upregulating MNX1-AS1 and slower by downregulating MNX1-AS1. Transfected MNX1-AS1 led to an increase in the number of invasion cells, whereas transfected sh-MNX1-AS1 resulted in a decrease in the number of invasion cells. Moreover, MNX1-AS1 affected the expression of EMT markers. These findings indicated that MNX1-AS1 could promote migration, invasion, and EMT to affect bladder cancer and might play critical roles in bladder cancer development.

Previous research showed that MNX1-AS1 regulated the expression of SEC61 translocon subunit alpha 1 through sponging miR-218-5p in colon adenocarcinoma, suggesting that MNX1-AS1/miR-218-5p axis is involved in colon adenocarcinoma progression (Ye et al., 2019). Nevertheless, the roles of MNX1-AS1/miR-218-5p axis in bladder cancer have not been studied. Here, we found that knockdown of MNX1AS1 suppressed proliferation, migration, invasion, and EMT of bladder cancer cells, which could be reversed by miR-218-5p inhibitor. These indicated that MNX1-AS1 could regulate proliferation, migration, invasion, and EMT of bladder cancer cells by sponging miR-218-5p.

RAB1A is a member of the small GTPases (Satoh et al., 2003). Recent evidence indicated that RAB1A is closely linked to human diseases, especially cancer (Shimada et al., 2005; Wang et al., 2016; Xu et al., 2017). RAB1A could function as an activator of mTORC1 in colorectal cancer (Thomas et al., 2014), prostate cancer (Sun et al., 2014), and hepatocellular carcinoma (Xu et al., 2015). These implied that RAB1A could influence downstream molecules of mTORC1, such as S6K1 (Zoncu et al., 2011; Cheng et al., 2019). A report has shown that RAB1A was related to p-S6K1 (Cheng et al., 2019). Our study showed that RAB1A was the target of miR-218-5p. Regulating miR-218-5p expression could modulate RAB1A expression, thereby affecting the activation of S6K1. Moreover, MNX1-AS1 could also regulate RAB1A expression by sponging miR-218-5p, thereby modulating the phosphorylation of $\mathrm{S} 6 \mathrm{~K} 1$ and cell proliferation, migration, invasion, and EMT abilities of bladder cancer cells. These suggested that MNX1-AS1 regulated RAB1A expression by sponging miR-218-5p to promote bladder cancer, and MNX1-AS1 may activate mTORC1 signaling to affect bladder cancer progression.

Furthermore, in vivo experiments confirmed that sh-MNX1-AS1 significantly promoted the expression of miR-218-5p and inhibited tumor growth, RAB1A expression, and S6K1 activation. These data suggested that MNX1-AS1/miR-218-5p/RAB1A axis could regulate bladder cancer progression in vivo.

\section{Conclusion}

The MNX1-AS1/miR-218-5p/RAB1A axis played an important role in bladder cancer. MNX1-AS1 could regulate the expression of RAB1A by sponging miR-218-5p, which contributed to the pathogenesis and progression of bladder cancer. 


\section{Authorship Contributions}

Participated in research design: Wang, Jia.

Conducted experiments: Wang, Xing, Nikzad, Liu, Y. Zhang.

Performed data analysis: Wang, Li.

Wrote or contributed to the writing of the manuscript: Wang, E. Zhang, Jia.

\section{References}

Brabletz T, Kalluri R, Nieto MA, and Weinberg RA (2018) EMT in cancer. Nat Rev Cancer 18:128-134.

Bray F, Ferlay J, Soerjomataram I, Siegel RL, Torre LA, and Jemal A (2018) Global cancer statistics 2018: GLOBOCAN estimates of incidence and mortality worldwide for 36 cancers in 185 countries. CA Cancer J Clin 68:394-424.

Chen H, Lin YW, Mao YQ, Wu J, Liu YF, Zheng XY, and Xie LP (2012) MicroRNA$449 \mathrm{a}$ acts as a tumor suppressor in human bladder cancer through the regulation of pocket proteins. Cancer Lett 320:40-47.

Cheng Y, Yang X, Deng X, Zhang X, Li P, Tao J, and Lu Q (2015) MicroRNA-218 inhibits bladder cancer cell proliferation, migration, and invasion by targeting BMI-1. Tumour Biol 36:8015-8023.

Cheng Z, Shao X, Xu M, Wang J, Kuai X, Zhang L, Wu J, Zhou C, and Mao J (2019) Rab1A promotes proliferation and migration abilities via regulation of the HER2/ AKT-independent mTOR/S6K1 pathway in colorectal cancer. Oncol Rep 41: $2717-2728$.

Ebrahimi H, Amini E, Pishgar F, Moghaddam SS, Nabavizadeh B, Rostamabadi Y, Aminorroaya A, Fitzmaurice C, Farzadfar F, Nowroozi MR, et al. (2019) Global, regional and national burden of bladder cancer, 1990 to 2016: results from the GBD study 2016. J Urol 201:893-901.

Ferro M, Vartolomei MD, Russo GI, Cantiello F, Farhan ARA, Terracciano D, Cimmino A, Di Stasi S, Musi G, Hurle R, et al. (2019) An increased body mass index is associated with a worse prognosis in patients administered BCG immunotherapy for T1 bladder cancer. World $J$ Urol 37:507-514.

Gao Y, Xu Y, Wang J, Yang X, Wen L, and Feng J (2019) lncRNA MNX1-AS1 promotes glioblastoma progression through inhibition of miR-4443. Oncol Res 27: 341-347.

Hao S, Yao L, Huang J, He H, Yang F, Di Y, Jin C, and Fu D (2018) Genome-wide analysis identified a number of dysregulated long noncoding RNA (lncRNA) in human pancreatic ductal adenocarcinoma. Technol Cancer Res Treat 17: 1533034617748429.

Li P, Yang X, Cheng Y, Zhang X, Yang C, Deng X, Li P, Tao J, Yang H, Wei J, et al. (2017) MicroRNA-218 increases the sensitivity of bladder cancer to cisplatin by targeting Glut1. Cell Physiol Biochem 41:921-932.

Li S, Xu X, Xu X, Hu Z, Wu J, Zhu Y, Chen H, Mao Y, Lin Y, Luo J, et al. (2013) MicroRNA-490-5p inhibits proliferation of bladder cancer by targeting c-Fos. Biochem Biophys Res Commun 441:976-981.

Liu H, Han L, Liu Z, and Gao N (2019a) Long noncoding RNA MNX1-AS1 contributes to lung cancer progression through the miR-527/BRF2 pathway. J Cell Physiol 234 13843-13850.

Liu X, Yang Q, Yan J, Zhang X, and Zheng M (2019b) LncRNA MNX1-AS1 promotes the progression of cervical cancer through activating MAPK pathway. J Cell Biochem 120:4268-4277.

Lv M, Zhong Z, Huang M, Tian Q, Jiang R, and Chen J (2017a) lncRNA H19 regulates epithelial-mesenchymal transition and metastasis of bladder cancer by miR-29b-3p as competing endogenous RNA. Biochim Biophys Acta Mol Cell Res 1864 $1887-1899$

Lv Y, Li H, Li F, Liu P, and Zhao X (2017b) Long noncoding RNA MNX1-AS1 knockdown inhibits cell proliferation and migration in ovarian cancer. Cancer Biother Radiopharm 32:91-99.

Ma QY, Li SY, Li XZ, Zhou TF, Zhao YF, Liu FL, Yu XN, Lin J, Chen FY, Cao J, et al (2019) Long non-coding RNA DILC suppresses bladder cancer cells progression. Gene 710:193-201.

Matboli M, Shafei AE, Ali MA, El-Din Ahmed TS, Naser M, Abdel-Rahman T, Anber N, and Ali M (2019) Role of extracellular LncRNA-SNHG14/miRNA-3940-5p/ NAP12 mRNA in colorectal cancer. Arch Physiol Biochem DOI: 10.1080/13813455. 2019.1650070 [published ahead of print].

Nieto MA, Huang RY, Jackson RA, and Thiery JP (2016) EMT: 2016. Cell 166: $21-45$.
Sassenberg M, Droop J, Schulz WA, Dietrich D, Loick SM, Wiek C, Scheckenbach K, Gaisa NT, and Hoffmann MJ (2019) Upregulation of the long non-coding RNA CASC9 as a biomarker for squamous cell carcinoma. BMC Cancer 19:806

Satoh A, Wang Y, Malsam J, Beard MB, and Warren G (2003) Golgin-84 is a rab1 binding partner involved in Golgi structure. Traffic 4:153-161.

Shimada K, Uzawa K, Kato M, Endo Y, Shiiba M, Bukawa H, Yokoe H, Seki N, and Tanzawa H (2005) Aberrant expression of RAB1A in human tongue cancer. $B r$ $J$ Cancer 92:1915-1921.

Sleeman JP and Thiery JP (2011) SnapShot: the epithelial-mesenchymal transition. Cell 145:162.e1.

Spano D, Heck C, De Antonellis P, Christofori G, and Zollo M (2012) Molecular networks that regulate cancer metastasis. Semin Cancer Biol 22:234-249.

Sun M and Kraus WL (2015) From discovery to function: the expanding roles of long noncoding RNAs in physiology and disease. Endocr Rev 36:25-64.

Sun T, Wang X, He HH, Sweeney CJ, Liu SX, Brown M, Balk S, Lee GS, and Kantoff PW (2014) MiR-221 promotes the development of androgen independence in prostate cancer cells via downregulation of HECTD2 and RAB1A. Oncogene 33: $2790-2800$

Thomas JD, Zhang YJ, Wei YH, Cho JH, Morris LE, Wang HY, and Zheng XF (2014) Rab1A is an mTORC1 activator and a colorectal oncogene. Cancer Cell 26:754-769. Wang KC and Chang HY (2011) Molecular mechanisms of long noncoding RNAs. Mol Cell 43:904-914.

Wang M, Guo C, Wang L, Luo G, Huang C, Li Y, Liu D, Zeng F, Jiang G, and Xiao X (2018) Long noncoding RNA GAS5 promotes bladder cancer cells apoptosis through inhibiting EZH2 transcription. Cell Death Dis 9:238.

Wang X, Liu F, Qin X, Huang T, Huang B, Zhang Y, and Jiang B (2016) Expression of Rab1A is upregulated in human lung cancer and associated with tumor size and $\mathrm{T}$ stage. Aging (Albany NY) 8:2790-2798.

Wong MCS, Fung FDH, Leung C, Cheung WWL, Goggins WB, and Ng CF (2018) The global epidemiology of bladder cancer: a joinpoint regression analysis of its incidence and mortality trends and projection. Sci Rep 8:1129.

Wu P, Zhang G, Zhao J, Chen J, Chen Y, Huang W, Zhong J, and Zeng J (2018) Profiling the urinary microbiota in male patients with bladder cancer in China. Front Cell Infect Microbiol 8:167.

Wu Y, Tian S, Chen Y, Ji M, Qu Y, and Hou P (2019) miR-218 inhibits gastric tumorigenesis through regulating Bmi-1/Akt signaling pathway. Pathol Res Pract 215:243-250.

Xu BH, Li XX, Yang Y, Zhang MY, Rao HL, Wang HY, and Zheng XF (2015) Aberrant amino acid signaling promotes growth and metastasis of hepatocellular carcinomas through Rab1A-dependent activation of mTORC1 by Rab1A. Oncotarget 6: 20813-20828.

Xu H, Qian M, Zhao B, Wu C, Maskey N, Song H, Li D, Song J, Hua K, and Fang L (2017) Inhibition of RAB1A suppresses epithelial-mesenchymal transition and proliferation of triple-negative breast cancer cells. Oncol Rep 37:1619-1626.

Xu Y, He Q, Lu Y, Tao F, Zhao L, and Ou R (2018) MicroRNA-218-5p inhibits cell growth and metastasis in cervical cancer via $L Y N / \mathrm{NF}-\kappa \mathrm{B}$ signaling pathway. Cancer Cell Int 18:198.

Xuan C, Jin M, Gao Y, Xu S, Wang L, Wang Y, Han R, and An Q (2019) miR-218 suppresses the proliferation of osteosarcoma through downregulation of E2F2. Oncol Lett 17:571-577.

Yang G, Lu W, Yu D, Sun C, Guo J, Li Z, and Guan F (2016) Quantitative analysis of differential proteome expression in epithelial-to-mesenchymal transition of bladder epithelial cells using SILAC method. Molecules 21:84.

Yang R, Wang L, and Han M (2018) MNX1-AS1 is a novel biomarker for predicting clinical progression and poor prognosis in lung adenocarcinoma. J Cell Biochem DOI: 10.1002/jcb.27996 [published ahead of print].

Ye Y, Gu B, Wang Y, Shen S, and Huang W (2019) E2F1-mediated MNX1-AS1-miR218-5p-SEC61A1 feedback loop contributes to the progression of colon adenocarcinoma. J Cell Biochem 120:6145-6153.

Yun SJ and Kim WJ (2013) Role of the epithelial-mesenchymal transition in bladder cancer: from prognosis to therapeutic target. Korean J Urol 54:645-650.

Zoncu R, Efeyan A, and Sabatini DM (2011) mTOR: from growth signal integration to cancer, diabetes and ageing. Nat Rev Mol Cell Biol 12:21-35.

Address correspondence to: Dr. Zhankui Jia, Department of Urology, The First Affiliated Hospital of Zhengzhou University, 1 East Jianshe Rd., Zhengzhou 450052, People's Republic of China. E-mail: jiazhankui@126.com 